\title{
On the properties of Ascent and Descent of Composition operator on Orlicz spaces
}

\author{
Ratan Kumar Giri *, Shesadev Pradhan \\ Dept. of Mathematics, National Institute of Technology, Rourkela, Odisha, India \\ e-mail: giri90ratan@gmail.com, pradhansh@nitrkl.ac.in
}

\begin{abstract}
Here, the composition operators on Orlicz spaces with finite ascent and descent as well as infinite ascent and descent are characterized.

Keywords: Orlicz function, Orlicz Space, Radon-Nikodym derivative, Composition operator.
\end{abstract}

2010 AMS Mathematics Subject Classification: 46E30; 46.35; $47 \mathrm{~B} 33$.

\section{Introduction and preliminaries}

Before going to start, let us recall the notion of ascent and descent of an operator on an arbitrary vector space $X$. So if $T: X \rightarrow X$ is an operator on $X$, then $N(T)$ and $R(T)$ denotes the null space and range space of $T$ respectively, that is

$$
N(T)=\{x \in X: T(x)=0\} \text { and } R(T)=\{T(x): x \in X\}
$$

The null space of $T^{k}$ is a $T$-invariant subspace of $X$, that is, $T\left(N\left(T^{k}\right)\right) \subseteq N\left(T^{k}\right)$ for every positive integer $k$. Indeed, if $x \in N\left(T^{k}\right)$ then $T^{k}(x)=0$ and therefore, $T^{k}(T(x))=T\left(T^{k}(x)\right)=0$, i.e., $T(x) \in N\left(T^{k}\right)$. We also have the following subspace inclusions:

$$
N(T) \subseteq N\left(T^{2}\right) \subseteq N\left(T^{3}\right) \subseteq \cdots
$$

The range $R\left(T^{k}\right)=T^{k}(X)$ of each operator $T^{k}$ is clearly another $T$-invariant subspace of $X$. Moreover:

$$
R(T) \supseteq R\left(T^{2}\right) \supseteq R\left(T^{3}\right) \supseteq \cdots
$$

Following definitions and well known results are relevant to our context ([1], [15], [19]);

${ }^{*}$ Corresponding author 
Theorem 1.1. For an operator $T: X \rightarrow X$ on a vector space we have have following:

1. If $N\left(T^{k}\right)=N\left(T^{k+1}\right)$ for some $k$, then $N\left(T^{n}\right)=N\left(T^{k}\right)$ for all $n \geq k$.

2. If $R\left(T^{k}\right)=R\left(T^{k+1}\right)$ for some $k$, then $R\left(T^{n}\right)=R\left(T^{k}\right)$ for all $n \geq k$.

We now introduce ascent and descent of an operator.

Definition 1.2. Let $T: X \rightarrow X$ be an operator on a vector space.

1. The ascent $\alpha(T)$ of $T$ is the smallest natural number $k$ such that $N\left(T^{k}\right)=$ $N\left(T^{k+1}\right)$. If there is no $k \in \mathbb{N}$ such that $N\left(T^{k}\right)=N\left(T^{k+1}\right)$, then we say that ascent $\alpha(T)$ is infinite.

2. Similarly, the descent $\delta(T)$ of $T$ is the smallest natural number $k$ such that $R\left(T^{k}\right)=R\left(T^{k+1}\right)$. If there is no $k \in \mathbb{N}$ such that $N\left(T^{k}\right)=N\left(T^{k+1}\right)$, then we say that descent $\delta(T)$ is infinite.

It turns out that if ascent and descent of an operator is finite then they are equal. This useful result stated next:

Theorem 1.3. If an operator $T: X \rightarrow X$ on a vector space has finite ascent and descent, then they must be coincide i.e., $\alpha(T)=\delta(T)=p<\infty$, and

$$
X=N\left(T^{p}\right) \oplus R\left(T^{p}\right)
$$

Moreover, if $x$ is a Banach space and $T$ is linear, then $R\left(T^{p}\right)$ is a closed subspace.

Let $T: X \rightarrow X$ be a bounded operator on the Banach space $X$. A pair $(V, W)$ of closed subspaces of $X$ is said to be a reducing pair of the operator $T$ if $X=V \oplus W$. Now we have the following result:

Theorem 1.4. A bounded operator $T: X \rightarrow X$ on a Banach space $X$ has finite ascent and descent if and only if $T$ has a reducing pair of closed subspaces $(V, W)$ such that the operator $T: V \rightarrow V$ is nilpotent and $T: W \rightarrow W$ is invertible.

Moreover, if $p=\alpha(T)=\delta(T)<\infty$, then the pair $(V, W)$ where $V=N\left(T^{p}\right)$ and $W=R\left(T^{p}\right)$ is the only reducing pair.

F. Riesz in [6] introduced the concept of ascent and descent for a linear operator in a connection with his investigation of compact linear operators. Also the study of ascent and descent has been done as a part of spectral properties of an operator ([3], [12]). Since the composition operator provide the diverse and illuminating example of operators which leads to study useful insight into structure theory of operators, it is desirable to study ascent and descent of these operators. In this paper, we study the ascent $\alpha(T)$ and descent $\delta(T)$, where $T$ is a composition operator on Orlicz Spaces. 


\section{Composition operator on Orlicz spaces}

Let $(\Omega, \Sigma, \mu)$ be a $\sigma$-finite complete measure space, where $\Sigma$ is a $\sigma$-algebra of subsets of an arbitrary set $\Omega$ and $\mu$ is a non-negative measure on $\Sigma$. Let $\phi:[0, \infty) \rightarrow[0, \infty)$ be non-decreasing continuous convex function such that $\phi(x)=0$ if and only if $x=0$ with $\lim _{x \rightarrow 0} \phi(x) / x=0$ and $\lim _{x \rightarrow \infty} \phi(x) / x=\infty$. Such a function $\phi$ is known as an Orlicz function. Let $L^{0}(\Omega)$ be denote the linear space of all equivalence classes of $\Sigma$-measurable functions on $\Omega$, where we identify any two functions are equal in the sense of $\mu$-almost everywhere on $\Omega$. Then the functional $I_{\phi}: L^{0}(\Omega) \rightarrow[0, \infty]$, defined by

$$
I_{\phi}(f)=\int_{\Omega} \Phi(f(t)) d \mu(t)
$$

where $f \in L^{0}(\Omega)$, is a pseudomodular [10], which is also defined as a modular in [8]. Let $L^{\phi}(\Omega)$ be the set of all measurable function such that $\int_{\Omega} \phi(\alpha|f|) d \mu<\infty$ for some $\alpha>0$. The space $L^{\phi}(\Omega)$ is called as Orlicz space and it is a Banach space with two norms: the Luxemberg norm [18], defined as

$$
\|f\|_{\phi}=\inf \left\{k>0: I_{\phi}\left(\frac{|f|}{k}\right) \leq 1\right\}
$$

and the Orlicz norm in the Amemiya form [7, 13] is given as

$$
\|f\|_{\phi}^{0}=\inf _{k>0}\left(1+I_{\phi}(k f)\right) / k .
$$

Note that the equality of the Orlicz norm and the Amemiya norm was proved in [7. If $\phi(x)=x^{p}, 1<p<\infty$, then $L^{\phi}(\Omega)=L^{p}$, the well known Banach space of $p$-integrable function on $\Omega$ with $\|f\|_{\phi}=\left(\frac{1}{p}\right)^{\frac{1}{p}}\|f\|_{p}([18])$. It is well known that $\|f\|_{\phi} \leq\|f\|_{\phi}^{0} \leq 2|| f \|_{\phi}$ and $\|f\|_{\phi} \leq 1$ if and only if $I_{\phi}(f) \leq 1$ ([4, 11]). Moreover, if $A \in \Sigma$ and $0<\mu(A)<\infty$, then $\left\|\chi_{A}\right\|_{\phi}=\frac{1}{\phi^{-1}\left(\frac{1}{\mu(A)}\right)}$, where $\chi_{A}$ is the characteristic function on $A$ (page no 78, [13]). For more literature concerning Orlicz spaces, we refer to Kufener, John and Fucik [2], Musielak [9] and Rao [13].

Let $\tau: \Omega \rightarrow \Omega$ be a measurable transformation, that is, $\tau^{-1}(A) \in \Sigma$ for any $A \in \Sigma$. If $\mu\left(\tau^{-1}(A)\right)=0$ for any $A \in \Sigma$ with $\mu(A)=0$, then $\tau$ is called as nonsingular. This condition implies that the measure $\mu \circ \tau^{-1}$, defined by $\mu \circ \tau^{-1}(A):=\mu\left(\tau^{-1}(A)\right)$ for $A \in \Sigma$, is absolutely continuous w.r.t $\mu\left(\mu \circ \tau^{-1} \ll \mu\right)$. Then the Radon-Nikodym theorem implies that there exist a non-negative locally integrable function $f_{\tau}(x)$ on $\Omega$ such that

$$
\mu \circ \tau^{-1}(A)=\int_{A} f_{\tau}(x) d \mu(t) \quad \text { for } A \in \Sigma .
$$

Any nonsingular measurable transformation $\tau$ induces a linear operator (Composition operator) $C_{\tau}$ from $L^{0}(\Omega)$ into itself which is defined as

$$
C_{\tau} f(t)=f(\tau(t)), \quad t \in \Omega, \quad f \in L^{0}(\Omega) .
$$


Here, the non-singularity of $\tau$ guarantees that the operator $C_{\tau}$ is well defined. Now, if the linear operator $C_{\tau}$ maps an Orlicz space $L^{\phi}(\Omega)$ into itself, then we call $C_{\tau}$ is a composition operator in $L^{\phi}(\Omega)$.

An Orlicz function $\phi$ is said to be satisfied the $\Delta_{2}$ condition if there exists a positive constant $K$ such that $\phi(2 x) \leq K \phi(x)$ for all $x>0$ ([11]). In [20] the necessary and sufficient condition about the boundedness and compactness of composition operators on Orlicz spaces are described. Regarding the boundedness of the composition operator $C_{\tau}$ from an Orlicz space $L^{\phi}(\Omega)$ into itself, we have the following theorem (Theorem $2.2,[20])$.

Theorem 2.1. The composition operator $C_{\tau}$ is bounded from an Orlicz space $L^{\phi}(\Omega)$ into itself if $\mu\left(\tau^{-1}(A)\right) \leq K \mu(A)$ holds for some $K>0$ and for all $A \in \Sigma$ with $\mu(A)<\infty$ and also converse holds when the Orlicz function $\phi$ satisfies $\Delta_{2}$ condition for all $x>0$.

Through out this paper, we assume that the composition operator is continuous. In [16, kernel of the Composition operator $C_{\tau}$ is obtained. It is shown that $N\left(C_{\tau}\right)=$ $L^{\phi}\left(\Omega_{\circ}\right)$, where $\Omega_{\circ}=\left\{x \in \Omega: f_{\tau}(x)=0\right\}$ and $L^{\phi}\left(\Omega_{\circ}\right)=\left\{f \in L^{\phi}(\Omega): f(x)=0\right.$ for $x \in$ $\left.\Omega \backslash \Omega_{0}\right\}$. For systematic study on composition operators on different spaces we refer to [5], [14] and [17].

Now, we will characterized the composition operator on Orlicz spaces $L^{\phi}(\Omega)$ with finite ascent and descent as well as infinite ascent and descent.

\section{Main Results}

Let us consider $\tau: \Omega \rightarrow \Omega$ be a nonsingular measurable transformation. Now $\tau$ is a non singular measurable transformation implies that $\tau^{k}$ is also non singular measurable transformation for every $k \geq 2$ with respect to the measure $\mu$. Hence $\tau^{k}$ is also induces a composition operator $C_{\tau^{k}}$. Note that for every measurable function $f, C_{\tau}^{k}(f)=$ $f \circ \tau^{k}=C_{\tau^{k}}(f)$. Also we have

$$
\cdots \ll \mu \circ \tau^{-(k+1)} \ll \mu \circ \tau^{-k} \ll \cdots \ll \mu \circ \tau^{-1} \ll \mu .
$$

Take $\mu \circ \tau^{-k}=\mu_{k}$. Then by Radon-Nikodym theorem, there exists a non-negative locally integrable function $f_{\tau^{k}}$ on $\Omega$ so that the measure $\mu_{k}$ can be represented as

$$
\mu_{k}(A)=\int_{A} f_{\tau^{k}}(x) d \mu(x), \text { for all } A \in \Sigma .
$$

The function $f_{\tau^{k}}$ is known as the Radon-Nikodym derivative of the measure $\mu_{k}$ with respect to the measure $\mu$. Now the following theorem characterized the composition operators with ascent $k$ : 
Theorem 3.1. The composition operator on Orlicz space $L^{\phi}(\Omega)$ has ascent $k \geq 1$ if and only if $k$ is the first positive integer such that the measures $\mu_{k}$ and $\mu_{k+1}$ are equivalent.

Proof. Suppose that $\mu_{k}$ and $\mu_{k+1}$ are equivalent. Then $\mu_{k+1} \ll \mu_{k} \ll \mu_{k+1}$. Since $\mu_{k} \ll \mu_{k+1} \ll \mu$, hence the chain rule of Radon-Nikodym derivative implies that

$$
\begin{aligned}
\frac{d \mu_{k}}{d \mu}(x) & =\frac{d \mu_{k}}{d \mu_{k+1}}(x) \cdot \frac{d \mu_{k+1}}{d \mu}(x) \\
\Rightarrow f_{\tau^{k}}(x) & =\frac{d \mu_{k}}{d \mu_{k+1}}(x) \cdot f_{\tau^{k+1}}(x)
\end{aligned}
$$

Similarly, $\mu_{k+1} \ll \mu_{k} \ll \mu$ implies that

$$
f_{\tau^{k+1}}(x)=\frac{d \mu_{k+1}}{d \mu_{k}}(x) \cdot f_{\tau k}(x)
$$

Now kernel of the $C_{\tau}^{k}$ given by $N\left(C_{\tau}^{k}\right)=N\left(C_{\tau^{k}}\right)=L^{\phi}\left(\Omega_{k}\right)$ where $\Omega_{k}=\{x \in \Omega$ : $\left.f_{\tau^{k}}(x)=0\right\}$ and $L^{\phi}\left(\Omega_{k}\right)=\left\{f \in \mathrm{E}^{\phi}(\Omega): f(x)=0\right.$ for $\left.x \in \Omega \backslash \Omega_{k}\right\}$. Similarly, $N\left(C_{\tau}^{k+1}\right)=L^{\phi}\left(\Omega_{k+1}\right)$, where $\Omega_{k+1}=\left\{x \in \Omega: f_{\tau^{k+1}}(x)=0\right\}$. Now from 3.2 and 3.3, it follows that $\Omega_{k}=\Omega_{k+1}$. Therefore we have,

$$
N\left(C_{\tau}^{k}\right)=L^{\phi}\left(\Omega_{k}\right)=L^{\phi}\left(\Omega_{k+1}\right)=N\left(C_{\tau}^{k+1}\right) .
$$

Since $k$ is the least hence, This shows that ascent of $C_{\tau}$ is $k$.

Conversely, suppose that ascent of $C_{\tau}$ is $k$. Now this implies that if $N\left(C_{\tau}^{k}\right)=L^{\phi}\left(\Omega_{k}\right)$ and $N\left(C_{\tau}^{k+1}\right)=L^{\phi}\left(\Omega_{k+1}\right)$, then $L^{\phi}\left(\Omega_{k}\right)=L^{\phi}\left(\Omega_{k+1}\right)$. Hence $\Omega_{k}=\Omega_{k+1}$ almost everywhere with respect to the measure $\mu$. So $\Omega_{k}=\left\{x \in \Omega: f_{\tau^{k}}(x)=0\right\}=\{x \in \Omega$ : $\left.f_{\tau k+1}(x)=0\right\}$. Now it is known that $\mu_{k+1} \ll \mu_{k}$. Only to show $\mu_{k} \ll \mu_{k+1}$. For this let $E \in \Sigma$ such that $\mu_{k+1}(E)=0$. Now we have the following cases:

Case-1: when $E \cap \Omega_{k}=\emptyset$

Then $0=\mu_{k+1}(E)=\int_{E} f_{\tau^{k+1}}(x) d \mu(x)$ implies that $\mu(E)=0$ as on $E, f_{\tau^{k+1}}(x)>0$. As $\mu_{k}(E)=\int_{E} f_{\tau^{k}}(x) d \mu(x)$ and $\mu(E)=0$, hence $\mu_{k}(E)=0$.

Case-2: when $E \cap \Omega_{k} \neq \emptyset$

Then we have,

$$
\begin{aligned}
0=\mu_{k+1}(E) & =\int_{E} f_{\tau^{k+1}}(x) d \mu(x) \\
& =\int_{E \backslash\left(E \cap \Omega_{k}\right)} f_{\tau^{k+1}}(x) d \mu(x)+\int_{E \cap \Omega_{k}} f_{\tau^{k+1}}(x) d \mu(x) \\
& =\int_{E \backslash\left(E \cap \Omega_{k}\right)} f_{\tau^{k+1}}(x) d \mu(x)
\end{aligned}
$$

Now this implies that $\mu\left(E \backslash\left(E \cap \Omega_{k}\right)\right)=0$. Therefore, in either case $\mu_{k+1}(E)=0$ implies that $\mu_{k}(E)=0$. Thus $\mu_{k+1} \ll \mu_{k} \ll \mu_{k+1}$. 
Corollary 3.2. Ascent of the composition operator $C_{\tau}$ on Orlicz spaces is infinite if and only if there does not exist any positive integer $k$ such that the measures $\mu_{k}$ and $\mu_{k+1}$ are equivalent.

We say that a measurable transformation $\tau$ is measure preserving if $\mu\left(\tau^{-1}(E)\right)=$ $\mu(E)$ for all $E \in \Sigma$. Then we have the following results:

Corollary 3.3. 1. If the measure $\mu$ is measure preserving then the ascent of the composition operator $C_{\tau}$ on Orlicz spaces $L^{\phi}(\Omega)$ is 1 .

2. If $\tau$ is a nonsingular surjective measurable transformation such that $\mu\left(\tau^{-1}(E)\right) \geq$ $\mu(E)$ for all $E \in \Sigma$, then also ascent of the composition operator induced by $\tau$ on Orlicz spaces is 1.

Note that for a non-singular surjective measurable transformation $\tau: \Omega \rightarrow \Omega$, measure of set a $A \subseteq \Omega$ positive does not always imply that the measure of $\tau^{-1}(A)$ is also positive. Example of one such measurable transformation is following:

Example 3.4. Consider unit interval $[0,1]$ with Lebesgue measure. Let $C$ be the Cantor set. Map $C$ onto $\left[0, \frac{1}{2}\right]$. For example convert ternary expansion to binary expansion and half it. Let the map be $S_{1}$. Next, map $[0,1] \backslash C$ onto $\left[\frac{1}{2}, 1\right]$ in a nonsingular way. In fact we can get a one-one bimeasurable map $S_{2}$ from $[0,1] \backslash C$ onto $\left[\frac{1}{2}, 1\right]$ such that measure of $S_{2}(A)$ equals half measure of $A$, for each $A$ in $[0,1] \backslash C$. Let $\tau$ be the map which is $S_{1}$ on $C$ and $S_{2}$ on $[0,1] \backslash C$.

This will satisfy our requirement. Here $\left[0, \frac{1}{2}\right]$ has positive measure but its inverse has measure zero. However if $B$ is a set of zero measure then measure of $\tau^{-1}(B)$ is same as measure of $S_{2}^{-1}(B)$ intersected with $[1 / 2,1]$.

Now the next result gives a necessary and sufficient condition for infinite ascent of the composition operator $C_{\tau}$ on Orlicz spaces $L^{\phi}(\Omega)$ in terms of range of $\tau$.

Theorem 3.5. Suppose that in the measure space $\Omega=(\Omega, \Sigma, \mu), \tau: \Omega \rightarrow \Omega$ is a nonsingular surjective measurable transformation such that if $\mu(A)>0$ then also $\mu\left(\tau^{-1}(A)\right)>0$, where $A \in \Sigma$. Then ascent of $C_{\tau}$ on Orlicz space $E^{\phi}(\Omega)$ is infinite if and only if there exists a sequence of subsets $\left\{\Omega_{k}\right\}$ of $\Omega$ such that for all $k \geq 1$

1. $0<\mu\left(\Omega_{k}\right)<\infty$

2. $\Omega_{k} \nsubseteq R\left(\tau^{k}\right)$ but $\Omega_{k} \subseteq R\left(\tau^{k-1}\right)$

3. $\mu\left(\Omega_{k} \cap R\left(\tau^{k}\right)\right)=0$ and $\mu\left(\Omega_{k} \cap \Omega_{k+1}\right)=0$. 
Proof. Assume that ascent of $C_{\tau}$ is infinite. Then $N\left(C_{\tau}^{k}\right) \neq N\left(C_{\tau}^{k+1}\right)$ for every $k \geq 0$. For $k=0$, this implies that there exists $f \neq 0$ a.e. in $\mathrm{七}^{\phi}(\Omega)$ such that $f \in N\left(C_{\tau}\right)$ i.e., $f \circ \tau=0$ a.e. . Take $\Omega_{\circ}=\{x \in \Omega: f(x)=0\}$. As $f \neq 0$, the set $\Omega \backslash \Omega_{\circ}=\Omega_{1}$ (say) is not empty. Since the measure $\mu$ is $\sigma$-finite, hence it has a subset of finite (positive) measure. Without loss of generality, we may assume that $0<\mu\left(\Omega_{1}\right)<\infty$.

Claim: $\Omega_{1} \nsubseteq R(\tau)$

If $\Omega_{1} \subseteq R(\tau)$, then $\tau\left(W_{1}\right)=\Omega_{1}$, where $W_{1}=\left\{x \in \Omega: \tau(x) \in \Omega_{1}\right\}$. Then for all $x \in W_{1}, f \circ \tau(x) \neq 0$ and by the given conation of $\tau, \mu\left(W_{1}\right)=\mu\left(\tau^{-1}\left(\Omega_{1}\right)\right)>0$ as $0<\mu\left(\Omega_{1}\right)<\infty$. This shows that $f \circ \tau \neq 0$ a.e. and $f \notin N\left(C_{\tau}\right)$, which is a contradiction.

Claim: $\mu\left(\Omega_{1} \cap R(\tau)\right)=0$

If $\Omega_{1} \cap R(\tau)=\emptyset$, then its obvious. If not, then $\Omega_{1} \cap R(\tau)=\Omega_{1}^{\prime}$ (say) is a proper subset of both $\Omega_{1}$ and $R(\tau)$ respectively. Then $\tau\left(W_{1}^{\prime}\right)=\Omega_{1}^{\prime}$, where $W_{1}^{\prime}=\{x \in \Omega$ : $\left.\tau(x) \in \Omega_{1}^{\prime} \subset \Omega_{1}\right\}$. Now if $\mu\left(\Omega_{1}^{\prime}\right)>0$, then by the given condition of $\tau$, implies that $\mu\left(W_{1}^{\prime}\right)=\mu\left(\tau^{-1}\left(\Omega_{1}^{\prime}\right)\right)>0$. But for all $x \in W_{1}^{\prime}, f \circ \tau(x) \neq 0$. This shows that $f \notin N\left(C_{\tau}\right)$, which is a contradiction. Therefore $\mu\left(\Omega_{1}^{\prime}\right)=0$.

Now for $k=1$, we have $N\left(C_{\tau}\right) \neq N\left(C_{\tau}^{2}\right)$. This implies that there exist $f \neq 0$ a.e. in $L^{\phi}(\Omega)$ such that $f \circ \tau^{2}=0$ a.e. but $f \circ \tau \neq 0$ a.e. . So if $\Omega_{2}^{\prime}=\{x \in \Omega: f \circ \tau(x) \neq 0\}$, then by the $\sigma$-finiteness of measure $\mu$, we can assume that $0<\mu\left(\Omega_{2}^{\prime}\right)<\infty$. Take $\Omega_{2}=\tau\left(\Omega_{2}^{\prime}\right)$. Then $\Omega_{2} \in R(\tau)$. As $\tau$ satisfies the given condition as above and $C_{\tau}$ is bounded, hence $0<\mu\left(\Omega_{2}\right)<\infty$. Now $\tau$ is nonsingular measurable transformation imply that $\tau^{2}$ also. Then by the similar kind of arguments it can be seen that $\Omega_{2} \nsubseteq R\left(\tau^{2}\right)$ and $\mu\left(\Omega_{2} \cap R\left(\tau^{2}\right)\right)=0$.

Therefore, we have two subsets $\Omega_{1}$ and $\Omega_{2}$ of finite measure with the following properties:

- $\Omega_{1} \subseteq R\left(\tau^{0}\right)=R(I)$ ( $I$ denote the identity map ) but $\Omega_{1} \nsubseteq R(\tau)$ and $\mu\left(\Omega_{1} \cap\right.$ $R(\tau))=0$

- $\Omega_{2} \subseteq R(\tau)$ but $\Omega_{2} \nsubseteq R\left(\tau^{2}\right)$ and $\mu\left(\Omega_{2} \cap R\left(\tau^{2}\right)\right)=0$

Now $\Omega_{1} \cap \Omega_{2}$ is a subset of $\Omega_{1} \cap R(\tau)$. As $\mu\left(\Omega_{1} \cap R(\tau)\right)=0$, hence $\mu\left(\Omega_{1} \cap \Omega_{2}\right)=0$. Hence by continuing similar process for every $k \geq 2$, we get a sequence $\left\{\Omega_{k}\right\}$ of subsets of finite measure such that $\Omega_{k} \nsubseteq R\left(\tau^{k}\right)$ but $\Omega_{k} \subseteq R\left(\tau^{k-1}\right)$ and $\mu\left(\Omega_{k} \cap R\left(\tau^{k}\right)\right)=0$ and $\mu\left(\Omega_{k} \cap \Omega_{k+1}\right)=0$.

Conversely, suppose that the given conditions are holds. As $0<\mu\left(\Omega_{k}\right)<\infty$, hence the characteristic function $\chi_{\Omega_{k}}$ is in Orlicz space $L^{\phi}(\Omega)$. As $\Omega_{k} \subseteq R\left(\tau^{k-1}\right)$ hence 
$\tau^{k-1}\left(W_{k}\right)=\Omega_{k}$, where $W_{k}=\left\{x \in \Omega: \tau^{k-1}(x) \in \Omega_{k}\right\}$. Then we have,

$$
\begin{aligned}
C_{\tau}^{k-1} \chi_{\Omega_{k}}\left(W_{k}\right) & =\chi_{\Omega_{k}}\left(\tau^{k-1}\left(W_{k}\right)\right) \\
& =\chi_{\Omega_{k}}\left(\Omega_{k}\right) \\
& =1
\end{aligned}
$$

This implies that $\chi_{\Omega_{k}} \notin N\left(C_{\tau}^{k-1}\right)$. But

$$
\begin{aligned}
C_{\tau}^{k} \chi_{\Omega_{k}}(\Omega) & =\chi_{\Omega_{k}}\left(\tau^{k}(\Omega)\right) \\
& =0 \text { a.e. }
\end{aligned}
$$

as $\Omega_{k} \nsubseteq R\left(\tau^{k}\right)=\tau^{k}(\Omega)$ and $\mu\left(\Omega_{k} \cap R\left(\tau^{k}\right)\right)=0$. Therefore, we have $\chi_{\Omega_{k}} \in N\left(C_{\tau}^{k}\right)$ but $\chi_{\Omega_{k}} \notin N\left(C_{\tau}^{k-1}\right)$. Since $k$ is arbitrary, hence the ascent of $C_{\tau}$ is infinite.

Remark 3.6. Here if we take our Orlicz function $\phi$ to be $x^{p}$ with $\Omega=\mathbb{N}$ and the measure $\mu$ is the counting measure, then the Orlicz space $L^{\phi}(\Omega)$ becomes the well known $l^{p}$ sequence spaces. Now suppose that $\tau: \mathbb{N} \rightarrow \mathbb{N}$ is onto. Then the measure $\mu$ satisfy previous criteria for $\tau$. Hence by the previous theorem we can say that "ascent of the composition operator $C_{\tau}$ on $l^{p}$ sequence space is infinite if and only if there exists a sequence of disjoints positive integers $\left\{n_{k}\right\}$ such that $n_{k} \notin R\left(\tau^{k}\right)$ but $n_{k} \in R\left(\tau^{k-1}\right)$ for each $k \geq 1 "$.

Now the following results are characterized the composition operators $C_{\tau}$ on Orlicz spaces $L^{\phi}(\Omega)$ with finite descent:

Theorem 3.7. If the map the map $\tau: R\left(\tau^{N}\right) \rightarrow R\left(\tau^{N}\right)$ is one-one for some $N$, then descent of composition operator is less than or equal to $N$.

Proof. Suppose that the map $\tau: R\left(\tau^{N}\right) \rightarrow R\left(\tau^{N}\right)$ is one-to-one. Let $f \in R\left(C_{\tau}^{N}\right)$. Then $f=C_{\tau}^{N}(g)$ for some $g \in L^{\phi}(\Omega)$. Now define the function

$$
h(x)= \begin{cases}g(y) ; & \text { if } y \in R\left(\tau^{N}\right) \quad \text { and } \quad \tau(y)=x \\ 0 ; & \text { otherwise }\end{cases}
$$

As $\tau: R\left(\tau^{N}\right) \rightarrow R\left(\tau^{N}\right)$ is one-to-one, hence the map $h$ is well defined and $g \in L^{\phi}(\Omega)$ implies that $h$ also in $L^{\phi}(\Omega)$. Note that $h \circ \tau(x)=g(x)$ for all $x \in R\left(\tau^{N}\right)$. Now we have,

$$
\begin{aligned}
h\left(\tau^{n+1}(x)\right) & =h\left(\tau\left(\tau^{N}(x)\right)\right. \\
& =g\left(\tau^{N}(x)\right) \\
& =f(x)
\end{aligned}
$$

This shows that $f \in R\left(C_{\tau}^{N+1}\right)$. Therefore, $R\left(C_{\tau}^{N}\right) \subseteq R\left(C_{\tau}^{N+1}\right)$. Hence this implies that descent of the composition operator is less than or equal to $N$ 
Corollary 3.8. If descent of composition operator is infinite then the map $\tau: R\left(\tau^{k}\right) \rightarrow$ $R\left(\tau^{k}\right)$ is not one-one for all $k \geq 0$.

Theorem 3.9. Assume that in the measure space $\Omega=(\Omega, \Sigma, \mu)$ every singleton set has positive measure. Then descent of composition operator is infinite if the map $\tau$ : $R\left(\tau^{k}\right) \rightarrow R\left(\tau^{k}\right)$ is not one-one for all $k \geq 0$.

Proof. Suppose that the map $\tau$ is not one-one. Then there exist $x_{1} \neq x_{2} \in R\left(\tau^{k}\right)$ such that $\tau\left(x_{1}\right)=\tau\left(x_{2}\right)$. Take $\Omega_{1}=\left\{x \in \Omega: \tau^{k}(x)=x_{1}\right\}$ and $\Omega_{2}=\left\{x \in \Omega: \tau^{k}(x)=x_{2}\right\}$. Note that $\Omega_{1} \cap \Omega_{2}=\emptyset$ and $\tau^{k+1}\left(\Omega_{1}\right)=\tau^{k+1}\left(\Omega_{2}\right)$. Now consider the function $f=$ $\chi_{\Omega_{1}}-\chi_{\Omega_{2}}$. As $0<\mu\left(\Omega_{1}\right)<\infty$ and $0<\mu\left(\Omega_{2}\right)<\infty$, hence $\chi_{\Omega_{1}}$ and $\chi_{\Omega_{2}}$ are belongs to Orlicz space $\left.L^{\phi} \Omega\right)$ and hence $f$ also in $L^{\phi}(\Omega)$. Take $g=\chi_{\left\{x_{1}\right\}}-\chi_{\left\{x_{2}\right\}}$. Then $g$ also in $L^{\phi}(\Omega)$ and $C_{\tau}^{k}(g)=f$. This implies that $f \in R\left(C_{\tau}^{k}\right)$. Now claim is that $f \notin R\left(C_{\tau}^{k+1}\right)$. If so then $f=C_{\tau}^{k+1}(g)$ for some $g \in L^{\phi}(\Omega)$. Then

$$
\begin{aligned}
1=f\left(\Omega_{1}\right) & =C_{\tau}^{k+1} g\left(\Omega_{1}\right) \\
& =g\left(\tau^{k+1}\left(\Omega_{1}\right)\right) \\
& =g\left(\tau^{k+1}\left(\Omega_{2}\right)\right) \\
& =f\left(\Omega_{2}\right) \\
& =-1,
\end{aligned}
$$

which is a contradiction. Hence we have $f \in R\left(C_{\tau}^{k}\right)$ but $f \notin R\left(C_{\tau}^{k+1}\right)$. Since $k$ is arbitrary, hence descent of $C_{\tau}$ is infinite.

Corollary 3.10. Suppose that the measure $\mu$ is as above. Then descent of the composition operator is $N$ if and only if there exists a natural number $N$ (smallest) such that the map $\tau: R\left(\tau^{N}\right) \rightarrow R\left(\tau^{N}\right)$ is one-one.

\section{Acknowledgement}

One of the authors (R. Kr. Giri) thanks the financial assistantship received from the Ministry of Human Resource Development (M.H.R.D.), Govt. of India.

\section{References}

[1] Angus E. Taylor, David C. Lay, Introduction to Functional Analysis, R.E. Krieger Publishing Company, 1980.

[2] A. Kufner, O. John and S. Fucik, Function Spaces, Academia Prague (1977).

[3] B. Aupetit, A Primer on Spectral Theory, Springer-verlag, Newyork, 1991. 
[4] B. S. Komal and S.Gupta, Composition operators on Orlicz spaces, Indian J. Pure Apply. Math., 32(2001), 1117-1122.

[5] E. Nordgren, Composition Operator On Hilbert Spaces, Lecture Notes on Mathematics, 693, 37-68, Springer-Verlag, Newyork, 1978.

[6] F. Riesz, Uber lineare Functionalgleichungen, Acta Math. 41 (1918), 71-98.

[7] H. Hudzik and L. Malingranda, Amemiya norm equals Orlicz norm in general, Indag. Math. N. S., 11(2000), 573-585.

[8] H. Nakano, Generalized modular spaces, Studia Math., 31(1968), 440-449.

[9] J. Musielak, Orlicz spaces and modular spaces, Lecture Notes in Math. 1034, Springer, Berlinn (1983).

[10] J. Musielak and W. Orlicz, On Modular Spaces, Studia math., 18(1959), 49-65.

[11] M. A. Krasnoselskii and Ya. B. Rutickii, Convex function and Orlicz spaces, Noordhorff, Groningen (1961).

[12] M. Burgos, A. Kaidi, M. Mbekhta and M. Oudghiri, The Descent Spectrum and Perturbations, J. Operator Theory, 56:2(2006), 259-271

[13] M. M. Rao and Z. D. Ren, Theory of Orlicz spaces, Marcel Dekker, New York (1991).

[14] R. K. Sing and J. S. Manhas, Composition operator on function spaecs, NorthHolland Mathematics Studies 179, Newyork (1993).

[15] Rajeev Kumar, Ascent and descent of weighted composition operators on $L^{p}$ spaces, Mathematicki Vensik, 60:1(2008), 47-51.

[16] Romesh Kumar, Composition operator on Oricz spaces, Integr. equ. Oper. Theory., 29(1997), 17-22.

[17] V. De. Cicoo and G. Marino, Composition Operator On Summable functions spaces, Le Mathematiche XLIV, (1989), 3-20.

[18] W. Luxemburg, Banach Function Spaces, Thesis, Technische Hogeschool te Delft, Netherland, 1955.

[19] Y. A. Abramovich and C. D. Aliprantis, An Invitation to Operator Theory, Graduate Studies in Mathematics 50, American Mathematical Society, 2002.

[20] Y. Cui, H. Hudzik, R. Kumar and L. Maligranda, Composition operators in Orlicz Spaces, J. Aust. Math. Soc., 76 (2004), 189-206. 